\title{
EXPERIMENTAL INVESTIGATION ON PROPERTIES OF RECYCLED AGGREGATES, BAGASSE ASH AND STEEL FIBER ON PROPERTIES OF CONCRETE
}

\author{
Laxman K.Lahamge ${ }^{1}$ \\ ${ }^{2}$ Department of Civil Engineering, Pravara Rural Engineering College, Loni \\ engg.laxman90@yahoo.com
}

\begin{abstract}
The use of recycled aggregates in concrete opens wide range of opportunities in the reuse of materials in the building industry. The use of recycled aggregates is a good remedial to the problem of an excess of waste material. . In fact, none of the results revealed the unsuitability of the recycled aggregates in concrete. This Work focuses on the possibility of the use of recycled aggregate concrete as a structural material with replacement level of 25\%,50\% and 75\% by weight of normal aggregates along with the hook end steel fiber used in this study varied from 0.5\% .1.0\%, and $1.5 \%$ by Volume of concrete. Bagasse ash is also used as a partial replacement of Portland cement with $5,10 \& 15 \%$ by weight of binder in order to improve the properties of RAC. The use of bagasse ash replace cement in RA concrete could improve compressive strength to be higher than that of concrete without bagasse ash at the same W/B ratio. This Work focuses on the possibility of the use of recycled aggregate concrete with bagasse ash and steel fibers as a structural material. The properties of concrete evaluated include compressive strength, Split tensile strength, flexural strength.
\end{abstract}

Keywords: Recycled Aggregates, Recycled Aggregate Concrete, Bagasse Ash Hook End Steel Fiber

\section{INTRODUCTION}

The conservation of natural resources is a basic need, which is directly connected with the survival of the human life. Parameters like environmental consciousness, protection of natural recourses, sustainable development play an important role in modern requirements for construction works. Globally, the concrete industry consumes large quantities of natural resources, which are becoming scarce to meet increasing demands. At the same time, many old buildings have reached the end of their service life and are being dismanteled, resulting in wasted materialand can be used as backfill material, and much being sent to landfills. Recycling leads to conservation of natural resources.

Bagasse Ash (BA) - Bagasse is a by-product of sugar industry and important fuel source for that industry. It is a fibrous with low density material with a very wide range of particle size distribution.

The sugarcane bagasse consists of approximately $50 \%$ of cellulose, $25 \%$ of hemicelluloses. Each ton of sugarcane generates approximately $25 \%$ of bagasse (at a moisture content of $50 \%$ ) and $0.62 \%$ of residual ash. The present study used ash collected from Asia's first co-operative sugar factory Padmashri Dr. Vitthalrao Vikhe Patil Co-operative
Sugar Factory, Pravaranagar with percentage replacement of $5 \%, 10 \%$ and $15 \%$ by weight of cement and mix design is done as per I.S.10262-2009.From many studies it is concluded that bagasse ash can be used as a cement replacing material because of its high silica content and fineness. It improves the workability of the fresh concrete.

Steel Fiber: Concrete is a brittle material as we know hence to improve ductility property and hence to introduce elasticity in concrete mass the steel fibers have been introduced in concrete.it improves many properties of concrete like long term strength,toughness and high sterss resistance. It has many applications in in structures such as flooring, housing, precast, tunneling, heavy duty pavement and mining.

Generally, aspect ratios of steel fibers used in concrete mix are varied between 50 to 100 .The most suitable volume fraction values for concrete mixes are between $0.5 \%$ to $1.5 \%$ by volume of concrete.

\section{EXPERIMENTAL PROGRAMME}

The quantity of ingredient materials and mix proportions as per design for M20 grade of concrete is as follow. 
Table 1. Identification Marks of in-gradient materials

\begin{tabular}{|c|c|c|c|c|}
\hline \multirow{2}{*}{$\begin{array}{c}\text { Sr. } \\
\text { No. }\end{array}$} & \multirow{2}{*}{ ID Mark } & \multicolumn{3}{|c|}{$\begin{array}{c}\text { Percentage Variation of } \\
\text { Ingradient Materials }\end{array}$} \\
\cline { 3 - 5 } & & RA\% & BA\% & SF\% \\
\hline 1 & RAC-A-1 & 25 & 5 & 0.5 \\
\hline 2 & RAC-A-2 & 25 & 10 & 1 \\
\hline 3 & RAC-A-3 & 25 & 15 & 1.5 \\
\hline 4 & RAC-B-1 & 50 & 5 & 0.5 \\
\hline 5 & RAC-B-2 & 50 & 10 & 1 \\
\hline 6 & RAC-B-3 & 50 & 15 & 1.5 \\
\hline 7 & RAC-C-1 & 75 & 5 & 0.5 \\
\hline 8 & RAC-C-2 & 75 & 10 & 1 \\
\hline 9 & RAC-C-3 & 75 & 15 & 1.5 \\
\hline
\end{tabular}

Table 2. Physical Properties of Ingredient Material of concrete.

\begin{tabular}{|c|c|c|c|c|}
\hline $\begin{array}{r}\mathrm{Sr} \\
\text { No. }\end{array}$ & Ingredient & \multicolumn{3}{|c|}{ Physical Property } \\
\hline \multirow[t]{4}{*}{1} & \multirow[t]{4}{*}{ Cement } & \multicolumn{2}{|c|}{ Fineness } & $4 \%$ \\
\hline & & \multirow{2}{*}{$\begin{array}{l}\text { Setting } \\
\text { Time(Min) }\end{array}$} & Initial & 63 \\
\hline & & & Final & 577 \\
\hline & & \multicolumn{2}{|c|}{ Standard Consistency } & $35 \%$ \\
\hline \multirow[t]{2}{*}{2} & \multirow{2}{*}{$\begin{array}{c}\text { Fine } \\
\text { Aggregates }\end{array}$} & \multicolumn{2}{|c|}{ Fineness Modulus } & 3.55 \\
\hline & & \multicolumn{2}{|c|}{ Specific Gravity } & 2.64 \\
\hline \multirow[t]{2}{*}{3} & \multirow{2}{*}{$\begin{array}{c}\text { Coarse } \\
\text { Aggregates }\end{array}$} & \multicolumn{2}{|c|}{ Fineness Modulus } & 4.71 \\
\hline & & \multicolumn{2}{|c|}{ Specific Gravity } & 2.70 \\
\hline \multirow[t]{2}{*}{4} & \multirow{2}{*}{$\begin{array}{c}\text { Recycled } \\
\text { Aggregates }\end{array}$} & \multicolumn{2}{|c|}{ Fineness Modulus } & 4.695 \\
\hline & & \multicolumn{2}{|c|}{ Specific Gravity } & 2.747 \\
\hline
\end{tabular}

Table 3. Slump Test Result

\begin{tabular}{|l|l|l|}
\hline $\begin{array}{l}\text { Sr. } \\
\text { No. }\end{array}$ & Id mark & $\begin{array}{l}\text { Slump } \\
(\mathrm{mm})\end{array}$ \\
\hline 1 & RAC-A-1 & 108 \\
\hline 2 & RAC-A-2 & 91 \\
\hline 3 & RAC-A-3 & 72 \\
\hline 4 & RAC-B-1 & 104 \\
\hline 5 & RAC-B-2 & 86 \\
\hline 6 & RAC-B-3 & 68 \\
\hline 7 & RAC-C-1 & 92 \\
\hline 8 & RAC-C-2 & 73 \\
\hline 9 & RAC-C-3 & 62 \\
\hline 10 & Normal & 120 \\
\hline
\end{tabular}

Table 4. Slump Vs \%Fiber Graph Details

\begin{tabular}{|l|l|l|l|}
\hline ID Marks & RAC-A & RAC-B & RAC-C \\
\hline RAC-1 & 108 & 104 & 92 \\
\hline RAC-2 & 91 & 86 & 73 \\
\hline RAC-3 & 72 & 68 & 62 \\
\hline
\end{tabular}

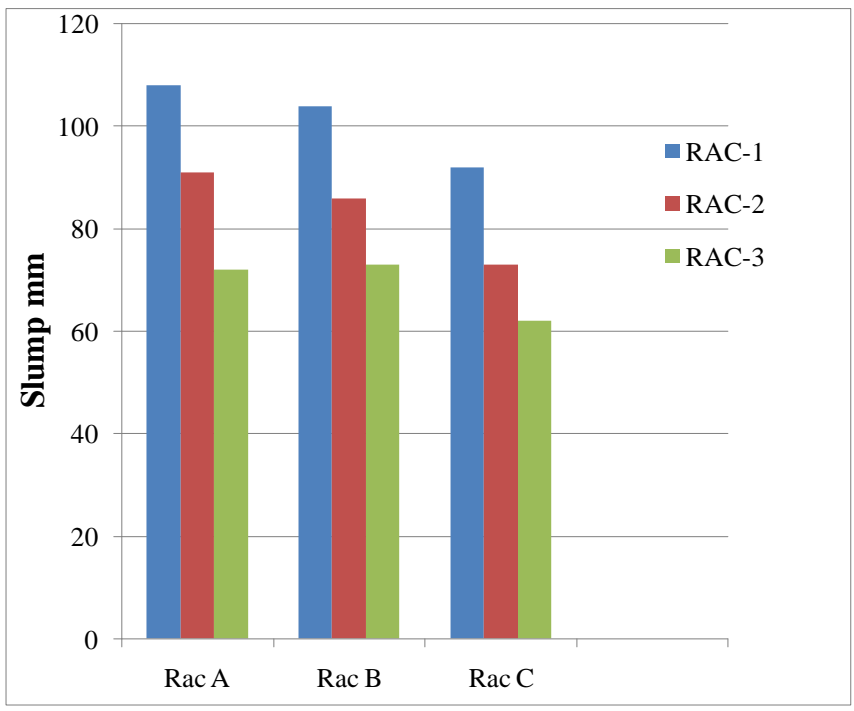

Graph 1. Slump Test Graph

Table 5. Proportion of Material used for M20 grade of concrete

Table 6. Average Comp. Strength of NC

\begin{tabular}{|c|c|c|c|c|}
\hline \multirow{2}{*}{ Sr no } & \multirow{2}{*}{ Id mark } & \multicolumn{4}{|c|}{$\begin{array}{r}\text { Average comp .strength } \\
\text { ( Mpa ) }\end{array}$} \\
\cline { 3 - 5 } & & 7 days & 28 days & 56 days \\
\hline 1 & NC & 18.81 & 26.72 & 29.92 \\
\hline
\end{tabular}

Table 7. Averages Compressive Strength of RAC

\begin{tabular}{|l|c|c|c|c|}
\hline $\begin{array}{l}\text { Sample } \\
\text { ID }\end{array}$ & $\begin{array}{l}\text { \% of fiber } \\
\text { No of days }\end{array}$ & $0.5 \%$ & $1.0 \%$ & $1.5 \%$ \\
\hline \multirow{3}{*}{ RAC-A } & 7 & 20.59 & 22.52 & 20.44 \\
\cline { 2 - 5 } & 28 & 23.55 & 25.77 & 22.52 \\
\cline { 2 - 5 } & 56 & 24.29 & 30.81 & 27.56 \\
\hline \multirow{3}{*}{ RAC-B } & 7 & 19.41 & 22.07 & 18.08 \\
\cline { 2 - 5 } & 28 & 24.74 & 23.56 & 20.15 \\
\hline \multirow{3}{*}{ RAC-C } & 56 & 29.78 & 24.29 & 23.56 \\
\cline { 2 - 5 } & 7 & 17.93 & 23.85 & 17.78 \\
\cline { 2 - 5 } & 28 & 22.37 & 27.56 & 20.89 \\
\hline
\end{tabular}

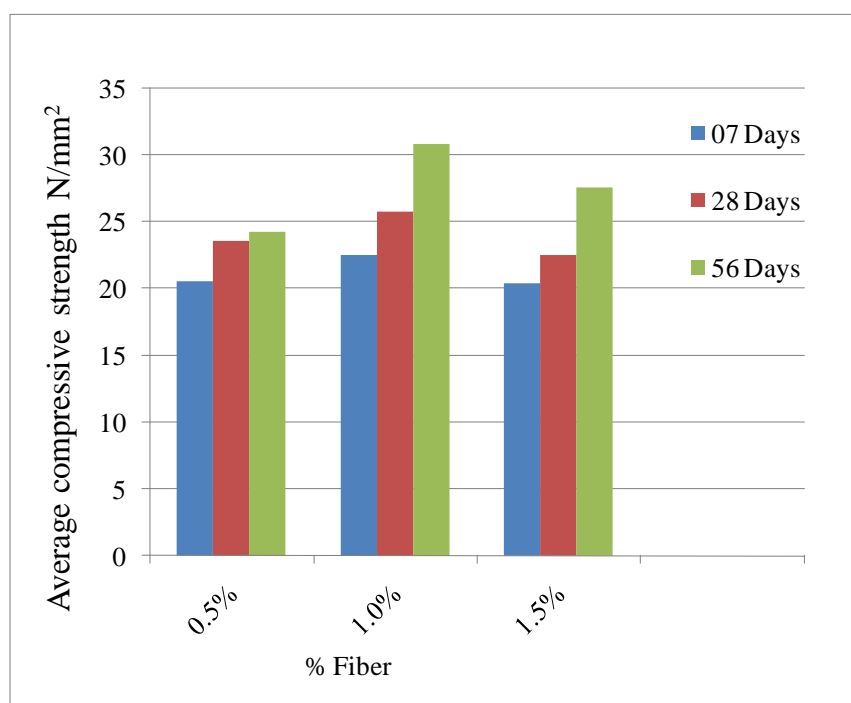

Graph 2- Average Compressive strength of RAC-A Vs Steel Fiber 


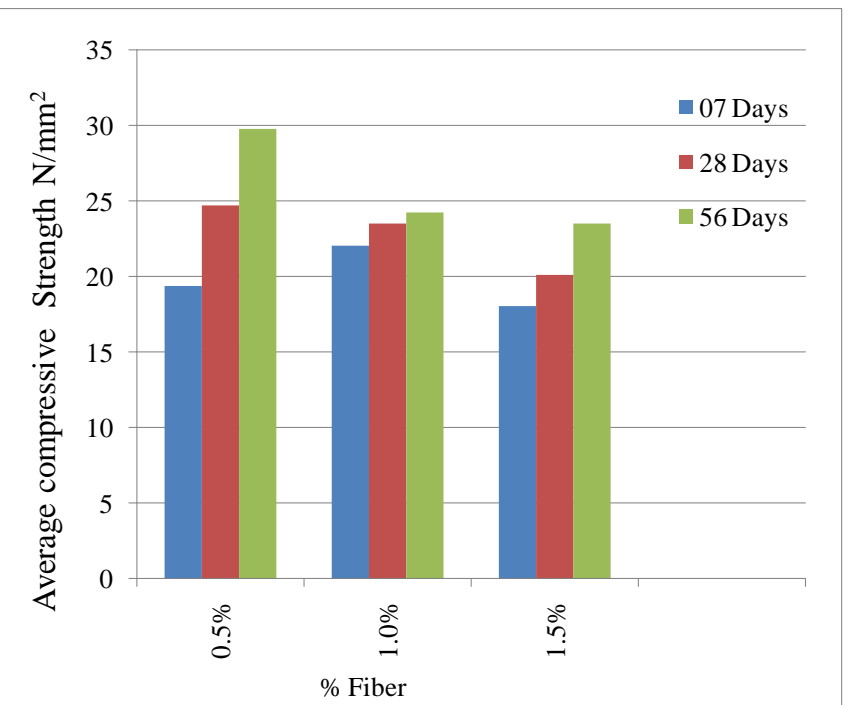

Graph 3- Average Compressive Strength of RAC-B Vs Steel Fiber

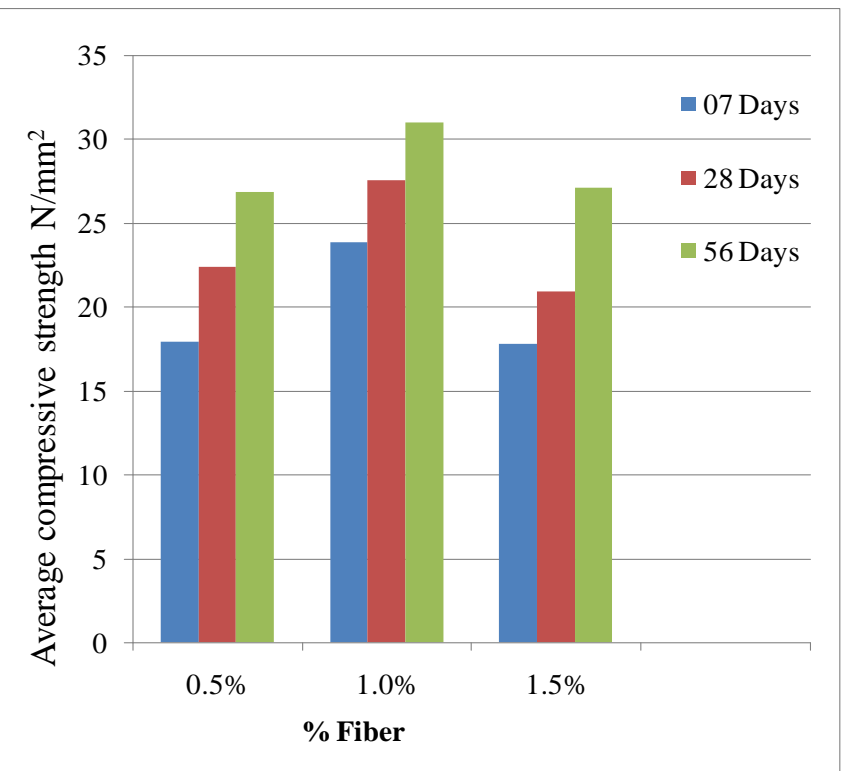

Graph 4- Average Compressive Strength of RAC-C Vs Steel Fiber

Table 8. Average Split Strength of NC

\begin{tabular}{|c|l|c|c|c|}
\hline $\begin{array}{c}\text { Sr } \\
\text { no }\end{array}$ & Id mark & \multicolumn{3}{|c|}{ Split Tensile strength ( Mpa ) } \\
\cline { 2 - 5 } & & 7 days & 28 days & 56 days \\
\hline 1 & NC & 1.50 & 3.11 & 3.06 \\
\hline
\end{tabular}

Table 9. Average Split Strength of RAC

\begin{tabular}{|c|c|c|c|c|}
\hline \begin{tabular}{|l} 
Sample \\
ID
\end{tabular} & $\begin{array}{l}\% \text { of fiber } \\
\text { No of days }\end{array}$ & $0.5 \%$ & $1.0 \%$ & $1.5 \%$ \\
\hline \multirow{3}{*}{ RAC-A } & 7 & 1.10 & 1.51 & 1.89 \\
\hline & 28 & 1.65 & 1.81 & 2.36 \\
\hline & 56 & 1.89 & 1.89 & 2.64 \\
\hline \multirow{3}{*}{ RAC-B } & 7 & 1.89 & 1.89 & 2.12 \\
\hline & 28 & 2.26 & 2.52 & 2.88 \\
\hline & 56 & 2.69 & 3.06 & 3.21 \\
\hline \multirow{3}{*}{ RAC-C } & 7 & 1.49 & 1.82 & 2.24 \\
\hline & 28 & 3.23 & 1.98 & 4.59 \\
\hline & 56 & 3.30 & 2.69 & 4.58 \\
\hline
\end{tabular}

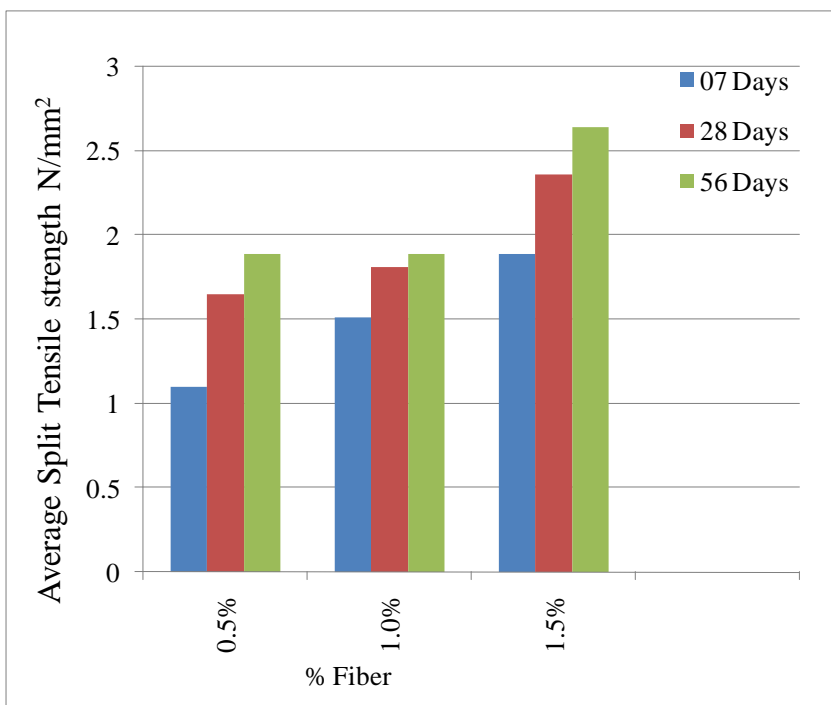

Graph 5 -Split Tensile Strength of RAC-A Vs Steel Fiber

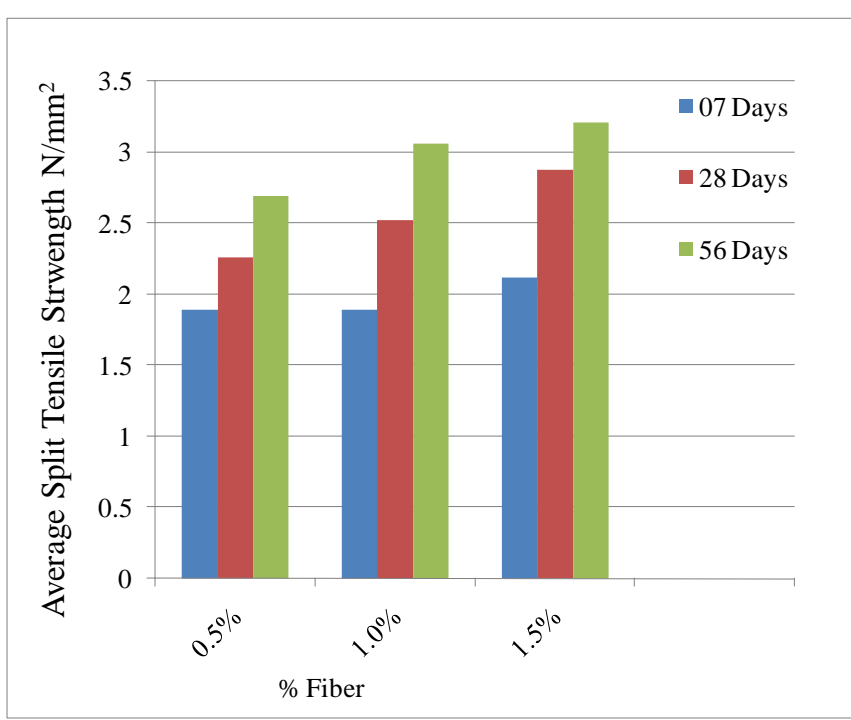

Graph 6- Split Tensile Strength of RAC-B Vs Steel Fiber

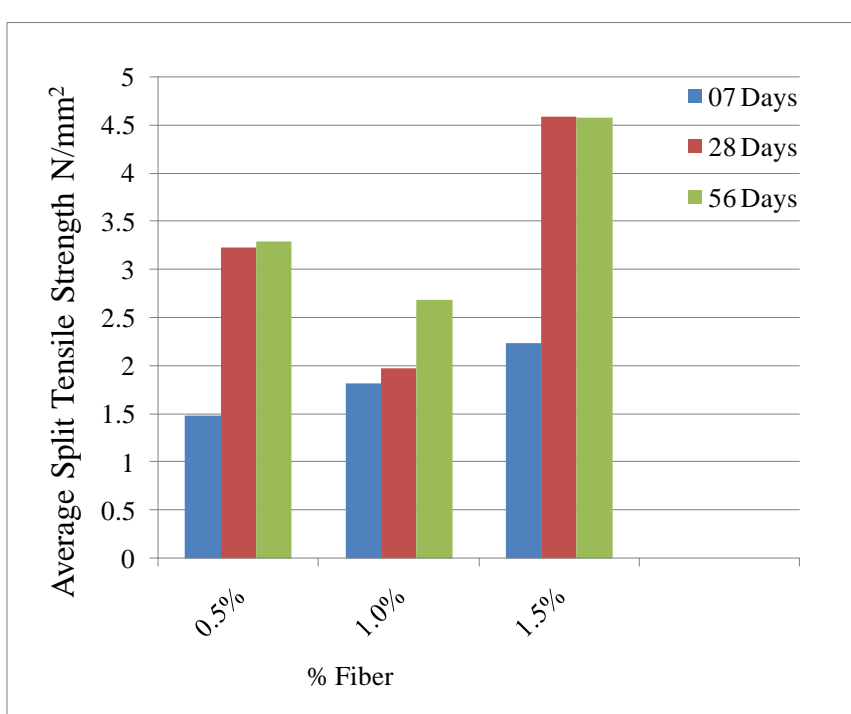

Graph 7- Split Tensile Strength of RAC-C Vs Steel Fiber 
Table 10. Average Flexural Strength of NC

\begin{tabular}{|c|l|c|c|c|}
\hline \multirow{2}{*}{$\begin{array}{c}\text { Sr. } \\
\text { No. }\end{array}$} & Id mark & \multicolumn{3}{|c|}{ Flexural strength ( Mpa ) } \\
\cline { 3 - 5 } & & 7 days & 28 days & 56 days \\
\hline 1 & NC & 4.49 & 5.55 & 5.83 \\
\hline
\end{tabular}

Table 11. Average Flexural Strength of RAC

\begin{tabular}{|l|c|c|c|c|}
\hline $\begin{array}{l}\text { Sample } \\
\text { ID }\end{array}$ & $\begin{array}{l}\% \text { of fiber } \\
\text { No of days }\end{array}$ & $0.5 \%$ & $1.0 \%$ & $1.5 \%$ \\
\hline \multirow{3}{*}{ RAC-A } & 7 & 5.58 & 4.87 & 5.05 \\
\cline { 2 - 5 } & 28 & 6.22 & 6.07 & 5.83 \\
\cline { 2 - 5 } & 56 & 6.53 & 6.35 & 6.89 \\
\hline \multirow{3}{*}{ RAC-B } & 7 & 2.95 & 4.53 & 5.63 \\
\cline { 2 - 5 } & 28 & 5.33 & 5.35 & 5.84 \\
\hline \multirow{3}{*}{ RAC-C } & 56 & 6.21 & 6.48 & 6.49 \\
\cline { 2 - 5 } & 7 & 3.50 & 3.91 & 4.93 \\
\cline { 2 - 5 } & 28 & 4.12 & 5.89 & 6.61 \\
\hline
\end{tabular}

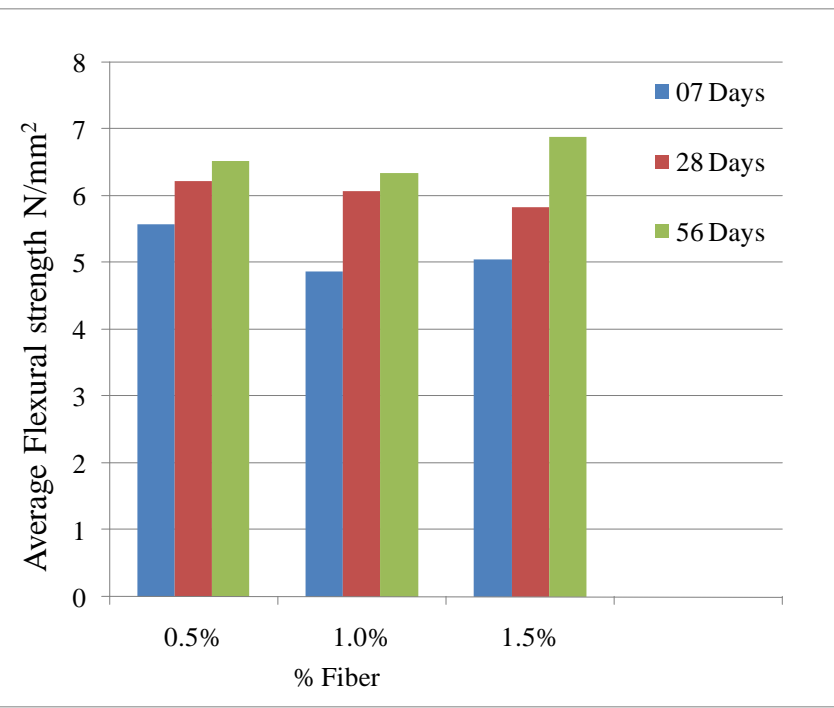

Graph 8. Flexural Strength of RAC-A Vs Steel Fiber

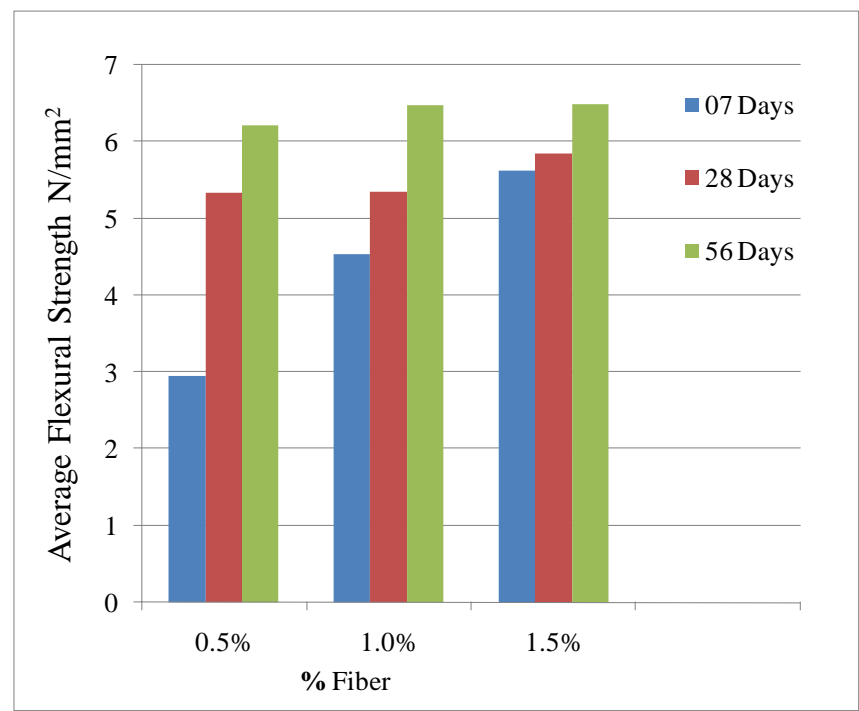

Graph 9. Flexural Strength of RAC-B Vs Steel Fiber

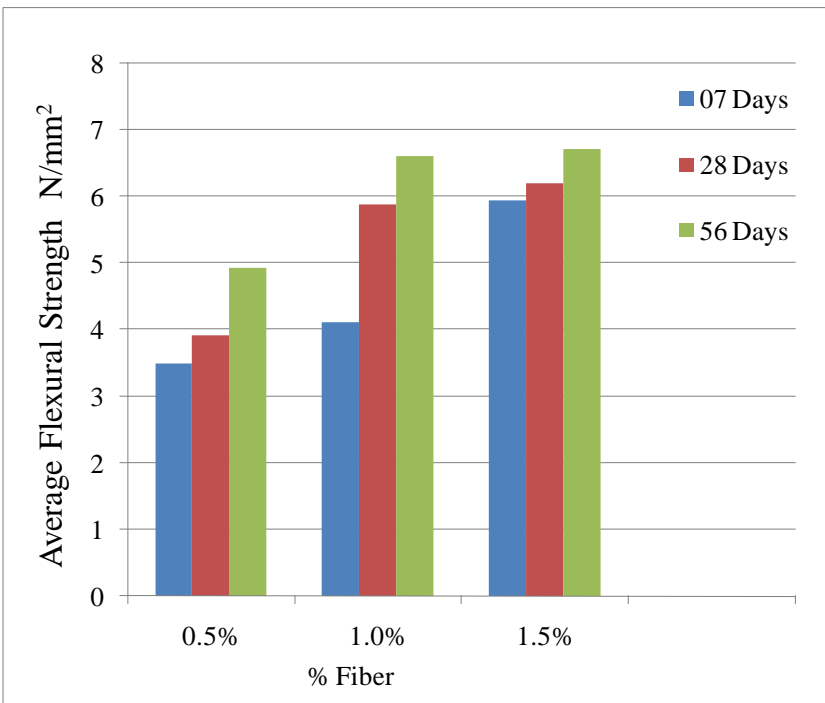

Graph 10. Flexural Strength of RAC-C Vs Steel Fiber

\section{CONCLUSION}

Following conclusion is drawn on the result discussed in the previous chapter :-

1. Compressive strength increases by $30.90 \%, 16.28 \%$ and $12.33 \%$ at 7,28 and 56 days respectively when 75 $\%$ RA , $10 \%$ BA and $1.0 \mathrm{SF}$ are used.

2. Split Tensile Strength increases by $55.55 \%, 80.0 \%$ and $38.78 \%$ at 7,28 and 56 days respectively when $75 \%$ RA, 15 BA and $1.5 \%$ SF are used.

3. Flexural strength increases by $35.30 \%, 21.92 \%$ and $41.95 \%$ at 7,28 and 56 days respectively when $75 \%$ RA , $5 \%$ BA and $0.5 \%$ SF are used.

4. As $\%$ of fiber increases the workability of concrete decreases which was observed from slump values but $0.5 \%$ fibers contents gives highest workability.

\section{REFERENCE}

[1]. Ajay Goyal, Hattori Kunio, Ogata Hidehiko, Mandula, "Properties and reactivity of sugarcane bagasse ash," Department of Environmental Engineering, United Graduate School of Agricultural Sciences, Tottori Universi, Tottori 680-8553, Japan, June 2010.

[3]. Amnon Katz, "Treatments for the Improvement of Recycled Aggregate", Journal of materials in civil engineering (ASCE), Vol.16, 2004, pp. 597-603.

[4]. C. J. Zega , Y. A. Villagra'n-Zaccardi , A. A. Di Maio, "Effect of natural coarse aggregate type on the physical and mechanical properties of recycled coarse aggregates", Materials and Structures, RILEM, Vol.43, 2010, pp. 195202.

[5]. D. Govindarajan and G. Jayalakshmi (2011) "XRD, FTIR and Microstructure Studies of Calcined Sugarcane Bagasse Ash", Advances in Applied Science Research, 2011, 2 (3):pp.544-549.

[8]. G. C. Cordeiro, R. D. Toledo Filho \& E. M. R. Fairbairn (2008),"Pozzolanic activity and filler effect of sugarcane bagasse ash in portland cement and lime mortars",Cement \& Concrete Composites, 30, (2008), pp.410-418. 
[9]. J.-Zh. Xiao, J.-B. Li, Ch. Zhang, "On relationships between the mechanical properties of recycled aggregate concrete: An overview", Materials and Structures, RILEM, Vol.39, May 2006, pp. 655-664.

[10]. K.Ganesan,K. Rajagopal,K.Thangavel (2007), "Evaluation of bagasse ash as supplementary cementitious material", Cement \& Concrete Composites, 30, May 2008,pp.410-418.

[11]. Keun-Hyeok Yang, Heon-Soo Chung, and Ashraf F, "Ashour, Influence of Type and Replacement Level of Recycled Aggregates on Concrete Properties".ACI Material Journal,Vol.105,No.3 May-June2008.

[12]. M. Chakradhara Rao, S. K. Bhattacharyya, S. V. Barai, "Influence of field recycled coarse aggregate on properties of concrete", Materials and Structures, RILEM, Vol.44, 2011, pp. 205-220.

[13]. M. Etxeberria A. R., Marı' E. Va'zquez, 'Recycled aggregate concrete as structural material", Materials and Structures, RILEM, Vol. 40, 2007, pp.529-541.

[14]. Marcos Oliverira De Paula (2009), "Sugarcane bagasse ash as a partial -portland-cement-replacement material", Dyna, year 77, Nro. 163, pp. 4754.Medellin, September, 2010. ISSN 00127353.

[15]. Mukesh Limbachiya, Mohammed Seddik Meddah, and Youssef Ouchagour "Performance of Portland/Silica Fume Cement ConcreteProduced with Recycled Concrete Aggregate".ACIMaterial Journalm,V.109,No.1,Jan-feb 2012.

[16]. Nik. D. Oikonomou, "Recycled concrete aggregates", Journal of Cement and Concrete Composites, Vol.27, 2005,pp. 315-318.

[17].Nuntachai Chusilp, Napongsatorn Likhitsripaiboon and Chai Jaturapitakkul, "Development of bagasse ash as a pozzolanic material in concrete", As. J. Energy Env. 2009, 10(03), pp .149-159.

[18]. P. Saravana Kumar and G. Dhinakaran, Ph.D, "Effect of Admixed Recycled Aggregate Concrete on Properties of Fresh and Hardened Concrete".Journal of material in Engineering-Vol.24,No.4,pp-494-498,April 12102.

19. R.Srinivasan and K.Sathiya, "Experimental study on bagasse ash in concrete"Vol.5, NO.2, pp.60-66, 2010.

[20].Shi Cong Kou, Chi Sun Poon, Dixon Chan, "Influence of Fly Ash as Cement Replacement on the Properties of Recycled Aggregate Concrete", Journal of materials in civil engineering (ASCE), Vol.19, June 2007, pp.709.

[21].S. Suvimol and C.Daungruedee (2008), Bagasse ash: "Effect of pozzolanic activity and application in cement use aspect", The 3rd ACF International Conference-ACF/VCA ,May 2008.

[22]. U.R.Kawade, V.R.Rathi, Vaishali D. Girge, "Effect of use of Bagasse Ash on Strength of Concrete" IJIRSETVol. 2, Issue 7, July 2013.

[23]. Wentao Zhang and Jason M. Ingham, M.ASCE, "Using Recycled ConcreteAggregates in New Zealand Ready-Mix Concrete Production", Journal of Materials In Civil Engineering (C Asce / MAY 2010 / Pp-443-450. 